\title{
Substrate Integrated Waveguide Triple-Passband Dual-Stopband Filter Using Six Cascaded Singlets
}

\author{
Mahbubeh Esmaeili and Jens Bornemann, Fellow, IEEE
}

\begin{abstract}
A triple-passband, dual-stopband filter consisting of six cascaded singlets is introduced in substrate integrated waveguide (SIW) technology. Each singlet produces one independent transmission zero (TZ). Four of six TZs are placed within the filter passband, producing three passbands separated by two stopbands. The corresponding coupling matrix is synthesized based on a combination of well-known analytic and optimization methods. The SIW filter is designed for stopbands centered at $\mathbf{1 0 . 2 5}$ and 11.23 $\mathrm{GHz}$, and passbands at $9.72,10.76$, and $11.76 \mathrm{GHz}$. The prototype is fabricated and measured. Good agreement between simulated and measured results is achieved.
\end{abstract}

Index Terms-Bandpass filter (BPF), coupling matrix, dual-bandstop filter, nonresonating node (NRN), substrate integrated waveguide (SIW).

\section{INTRODUCTION}

B ANDPASS filters (BPFs) have been investigated widely during the last two decades. But the design and fabrication of their counterparts, bandstop filters, have attracted less attention despite their important roles in modern wireless communication systems.

The traditional realization method of bandstop filters relies on loading a waveguide or transmission line with a group of series stopband stubs which are a quarter wavelength long and spaced by odd multiples of a quarter wavelength at the center frequency [1]. No control of reflection zeros (RZs), usually large physical dimensions and the first higher order mode appearance at three times the fundamental resonant frequency are disadvantages of this approach. A modified method, presented in [2], describes how to increase the first higher order resonant frequency to much higher multiples of the fundamental stopband frequency.

The flexible extracted pole technique allows the designer to synthesize both symmetric and asymmetric responses with arbitrarily located transmission zeros (TZs) by separating the in-line resonator by constant phase shifts [3].

A common approach to combine bandpass and bandstop characteristics is to utilize dual-behavior resonators (DBRs) [4]. However, they are difficult to realize in H-plane substrate integrated waveguide (SIW) technology.

Manuscript received October 29, 2013; revised January 12, 2014; accepted February 11, 2014. Date of publication June 05, 2014; date of current version June 20,2014. This work was supported by the TELUS Research Grant in Wireless Communications.

The authors are with the Department of Electrical and Computer Engineering, University of Victoria, Victoria, BC V8W 2Y2, Canada (e-mail: mesmaei@uvic.ca; j.bornemann@ieee.org).

Color versions of one or more of the figures in this paper are available online at http://ieeexplore.ieee.org.

Digital Object Identifier 10.1109/LMWC.2014.2316242
Bandstop filters can also be realized by coupled bandpass resonators [5], and the coupling matrix of such components can be synthesized by a method very similar to that of BPFs [5], [6]. Though this method enables us to create both elliptic and quasi-elliptic bandstop filters, the required direct and cross couplings might be too large to be realized. Moreover, source-load coupling is a necessity which places restrictions on the overall filter topology.

More recently, the nonresonating node (NRN) concept has been introduced to generate compact BPFs [7], [8]. Beside compactness, NRNs create direct paths that are bypassing the resonators, thus enabling us to produce $N$ TZs with $N$ resonant elements in an in-line configuration, i.e., without the need for source-load coupling.

Some single-band multi-resonator passband filters based on NRNs and in E-plane waveguides are reported in [7], but they are not using cascaded singlets. NRNs have been used in singlets and cascaded singlets to realized single-band BPFs in E-plane waveguide technology and two-layer microstrip configuration [8].

Two different configurations of band-reject filters based on NRNs have been reported in [9]. In one of them, the NRNs have been used with inverters, and in a second case, the NRNs are used with phase shifters and inverters. Both symmetric and nonsymmetric responses with arbitrary TZs can be realized. Microstrip and E-plane waveguide band-reject filters have been designed based on the methods proposed in [9].

In comparison with the other papers using the NRN concept to realize bandpass or bandstop filters, and we mentioned some of them [7]-[9], this is the first time that six cascaded singlets, in the form of $\mathrm{H}$-plane waveguides, are used to realize a wideband multiband triple passband and double stopband filter in SIW technology that allows us to control the locations of the stopband TZs as well as the passband RZs. Note that previously reported multiband bandpass SIW filters, like the one in [10], are based on coupled bandpass resonators without involving NRNs.

\section{Design Procedure}

As described in [8], each singlet can produce one $\mathrm{TZ}$ as well as one RZ. This is schematically shown in Fig. 1(a). Since SIW prototyping is envisioned, the $\mathrm{TE}_{201}$ mode is used as resonance while the $\mathrm{TE}_{10}$ mode creates the bypass coupling, thus producing one TZ. Using this concept, to create the triple-passband, dual-stopband filter, we first design a $13 \times 13$ coupling matrix (not shown here) based on Fig. 1(b). This coupling matrix is synthesized based on well-known analytic and optimization methods described in [6] and [11], with some modification to take into account the NRNs. Two stopband center frequencies are selected as 10.25 and $11.23 \mathrm{GHz}$, with bandwidths of 


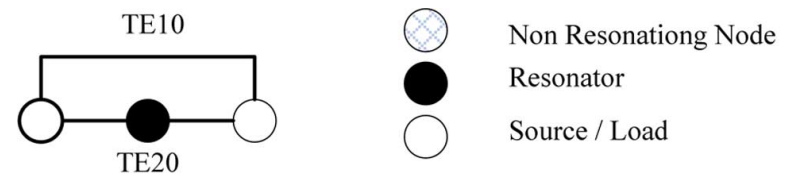

(a)

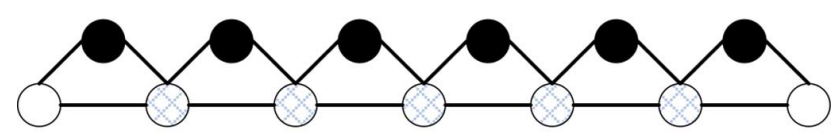

(b)

Fig. 1. Configuration of a singlet (a), and a passband filter consisting of six singlets (b).

$0.32 \mathrm{GHz}$ and $0.2 \mathrm{GHz}$, respectively. The center frequencies of the three passbands are $9.72,10.76$, and $11.76 \mathrm{GHz}$ with bandwidths of $0.39 \mathrm{GHz}, 0.37 \mathrm{GHz}$, and $0.39 \mathrm{GHz}$, respectively. The second step is to design a six-pole BPF by cascading six singlets as shown in Fig. 1(b), using the commercial software package $\mu$ Wave Wizard. The center frequency, bandwidth and return loss of such a wideband filter are $10.75 \mathrm{GHz}, 2.35 \mathrm{GHz}$, and $23 \mathrm{~dB}$, respectively. As expected this structure produces six TZs that can now be almost independently moved to the left or right side of the passband, or even into the passband. Each singlet is responsible for one TZ. The location of each TZ depends on the relative locations of the input and output apertures of its corresponding singlets [7]. Any changes in the input or output apertures size and location has also a very small effect on the other singlets, but it is not very significant and some fine optimization can eliminate these effects. This feature can be used to change the wideband passband filter to a triple-passband, double-stopband filter.

In the third step, the designed six-pole wideband BPF is optimized based on the coupling matrix synthesized in the first step to realize a dual-stopband, triple-passband filter. For use in a planar application, all filter dimensions are translated to a SIW model, using the method described in [12]. Finally, the frequency-domain solver of CST is used to simulate and fine-optimize the SIW filter shown in Fig. 2. The substrate is RT/Duroid 6002 with dielectric constant of 2.94 , thickness of $0.508 \mathrm{~mm}$

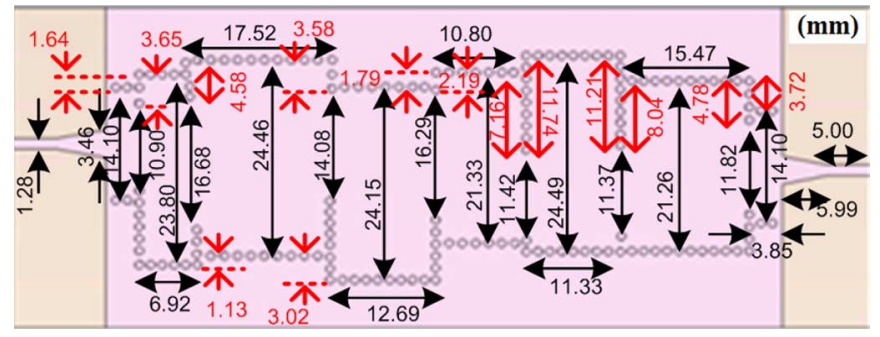

Fig. 2. Layout of the triple-passband dual-stopband filter with coaxial ports and microstrip to SIW transitions.

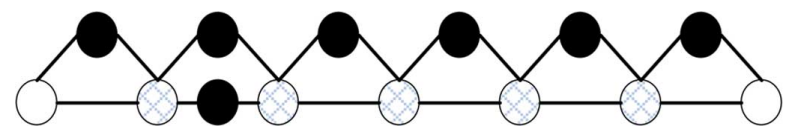

Fig. 3. Updated coupling scheme of the dual-band bandstop filter designed in Fig. 2.

and dielectric loss tangent of 0.0012 . The diameter of all via holes is $1 \mathrm{~mm}$ and the cutoff frequency of the input waveguide is $6.5 \mathrm{GHz}$. All dimensions of the filter are shown in Fig. 2, with distances measured between via centers.

During the optimization process, the length of the second resonator was increased which causes the fundamental $\mathrm{TE}_{102}$ mode to start resonating in this resonator in addition to the $\mathrm{TE}_{201}$ mode. To include this resonance, the filter coupling configuration in Fig. 1(b) is updated in Fig. 3. So we synthesize a new $14 \times 14$ coupling matrix and continue the optimization of the physical dimensions of the filter based on the new coupling matrix.

The normalized coupling matrix of the updated topology shown in Fig. 3 is presented in (1) at the bottom of this page. The scattering parameters corresponding to this coupling matrix are plotted in Fig. 4. Good agreement between simulated scattering parameters and those calculated from the coupling matrix is observed, especially in terms of TZs and RZs locations. Since the coupling matrix elements are assumed frequency-independent, it is difficult to reach the same return loss and attenuation in all passbands and stopbands, respectively. The simulation includes the full-wave frequency-dependent response of the filter. Therefore, the simulated scattering parameters show

1.3865
0.3772
0.6685
-0.4119
0.9886
0
0
0
0
0
0
0
0
0

$\begin{array}{cc}0 & 0 \\ 0 & 0 \\ -0.4119 & 0.9886 \\ -0.7181 & 0 \\ 0 & 0.2976 \\ -0.3733 & 0.4316 \\ 0 & 0 \\ 0 & 0 \\ 0 & 0 \\ 0 & 0 \\ 0 & 0 \\ 0 & 0 \\ 0 & 0 \\ 0 & 0\end{array}$

$\begin{array}{cc}0 & 0 \\ 0 & 0 \\ 0 & 0 \\ -0.3733 & 0 \\ 0.4316 & 0 \\ -0.3177 & -0.7379 \\ -0.7379 & -1.4926 \\ 0.6762 & 0.1423 \\ 0 & 0 \\ 0 & 0 \\ 0 & 0 \\ 0 & 0 \\ 0 & 0 \\ 0 & 0\end{array}$

$\begin{array}{cc}0 & 0 \\ 0 & 0 \\ 0 & 0 \\ 0 & 0 \\ 0 & 0 \\ 0.6762 & 0 \\ 0.1423 & 0 \\ -0.1711 & 0.3045 \\ 0.3045 & -0.452 \\ 0.8229 & 0.2532 \\ 0 & 0 \\ 0 & 0 \\ 0 & 0 \\ 0 & 0\end{array}$

0
0
0
0
0
0
0
0.8229
0.2532
-0.4707
-0.2082
0.6983
0
0

$\begin{array}{cc}0 & 0 \\ 0 & 0 \\ 0 & 0 \\ 0 & 0 \\ 0 & 0 \\ 0 & 0 \\ 0 & 0 \\ 0 & 0 \\ 0 & 0 \\ -0.2082 & 0.6983 \\ 0.0931 & 0.4429 \\ 0.4429 & -0.282 \\ 0 & -0.2948 \\ 0 & 1.2374\end{array}$

$\left.\begin{array}{cc}0 & 0 \\ 0 & 0 \\ 0 & 0 \\ 0 & 0 \\ 0 & 0 \\ 0 & 0 \\ 0 & 0 \\ 0 & 0 \\ 0 & 0 \\ 0 & 0 \\ 0 & 0 \\ -0.2948 & 1.2374 \\ 0.3563 & 0.3727 \\ 0.3727 & 0\end{array}\right]$




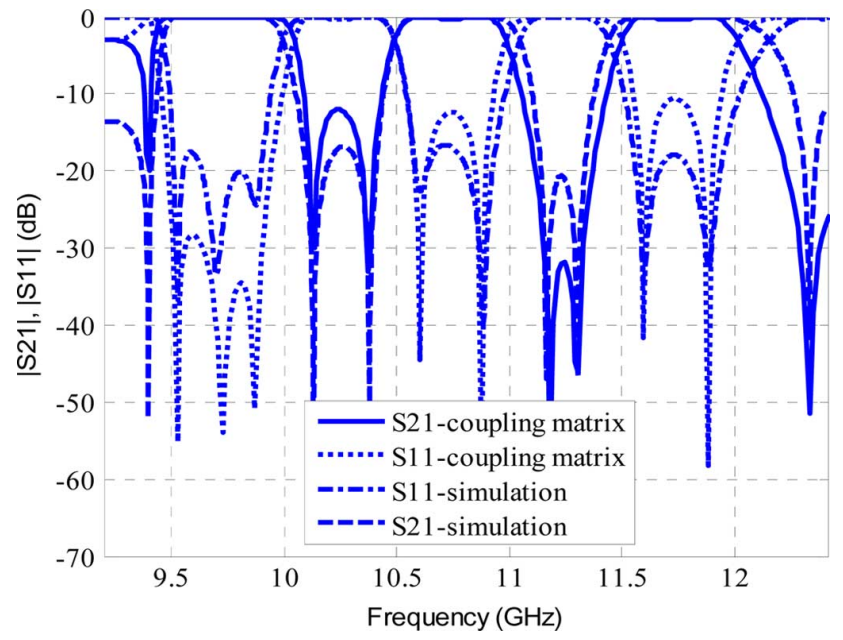

Fig. 4. Scattering parameters of the triple-passband dual-stopband filter; simulated response (CST) versus coupling matrix (M).

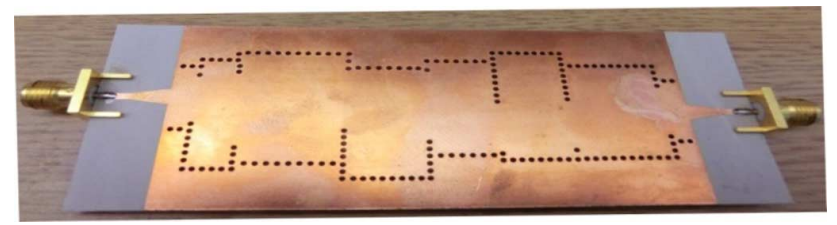

Fig. 5. The dual-band bandstop filter fabricated in SIW technology with microstrip ports and microstrip-to-SIW transitions.

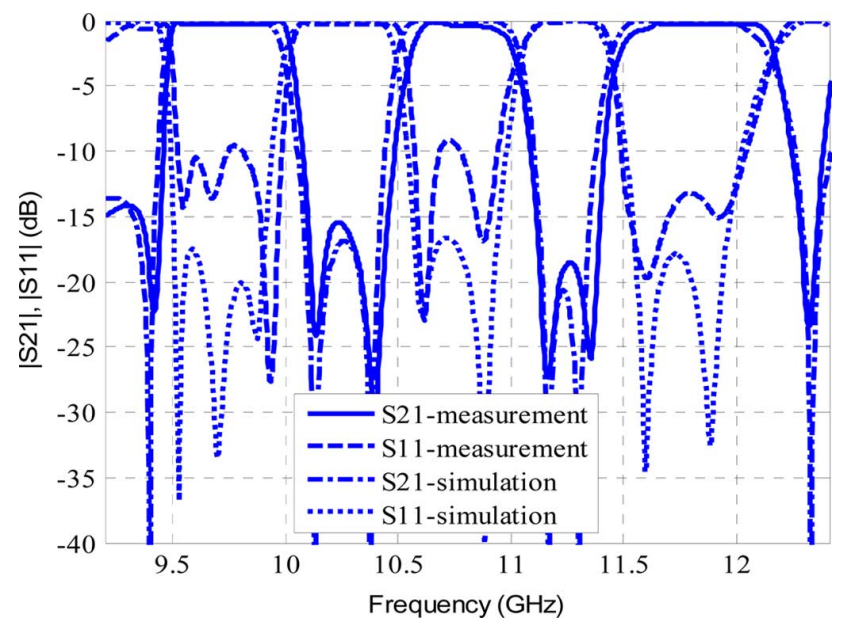

Fig. 6. Comparison between measured and simulated scattering parameters of the triple-passband, dual-stopband filter.

better passband return losses and stopband attenuations compared to scattering parameters calculated from the coupling matrix.

\section{FABRICATION AND MEASUREMENT}

The triple-passband, dual-stopband filter has been prototyped as shown in Fig. 5. Measurements were carried out with a vector network analyzer and Thru-Reflect-Line (TRL) calibration standards, which ideally calibrate out the SMA-to microstrip and microstrip-to-SIW transitions.

The measured results are compared with simulated responses in Fig. 6. Good agreement between simulated and measured transmission coefficients is achieved, especially with respect to the positions of the TZs and RZs. The measured passband return losses are $9.63 \mathrm{~dB}, 9.17 \mathrm{~dB}$, and $13.22 \mathrm{~dB}$, with minimum insertion losses of $0.33 \mathrm{~dB}, 0.45 \mathrm{~dB}$ and $0.3 \mathrm{~dB}$, respectively. The measured minimum attenuations in the two stopbands are 15.48 and $18.56 \mathrm{~dB}$ which agree reasonably well with simulations. The slight upward frequency shift is well within the limit of the substrate's permittivity of $2.94 \pm 0.04$. Moreover, the vias appear to have been drilled slightly larger than the nominal value of $1 \mathrm{~mm}$. That reduces the effective resonator widths and length, and thus the filter shifts upward in frequency (not shown here). The remaining differences, especially in return loss levels, are attributed to the differences in soldering coaxial connectors to the calibration standards and filter structure.

\section{CONCLUSION}

An approach for creating a dual-stopband filter within a wide passband is presented. Using cascaded singlets and the NRN concept, an SIW triple-passband, dual-stopband filter is designed and prototyped. The synthesized coupling matrix and measured results are in good agreement with simulations which validates the reliability of the design procedure. Measurements verify the simulated performance in principle. Some discrepancies, especially in the passband return loss, are attributed to the fabricated TRL calibration standards. Note that this approach can be extended to create multiple bandstop as well as BPFs since the locations of RZs and TZs as well as the center frequency and bandwidth of each stopband can be independently controlled.

\section{REFERENCES}

[1] G. L. Matthaei, L. Young, and E. M. T. Jones, "Bandstop filters," in Microwave Filters, Impedance-Matching Networks, and Coupling Structure. New York: McGraw-Hill, 1985, pp. 725-740.

[2] R. Levy, R. V. Snyder, and S. Shin, "Bandstop filters with extended upper passbands," IEEE Trans. Microw. Theory Tech., vol. 54, pp. 2503-2515, Jun. 2006.

[3] I. R. Rhodes and R. J. Cameron, "General extracted pole synthesis technique with application to low-loss $\mathrm{TE}_{011}$-mode filters," IEEE Trans. Microw. Theory Tech., vol. 28, pp. 1018-1028, Sep. 1980.

[4] C. Quendo, E. Rius, and C. Person, "Narrow bandpass filters using dual-behavior resonators," IEEE Trans. Microw. Theory Tech., vol. 51, pp. 734-743, Mar. 2003.

[5] S. Amari and U. Rosenberg, "Direct synthesis of a new class of bandstop filters," IEEE Trans. Microw. Theory Tech., vol. 52, pp. 607-615, Feb. 2004.

[6] R. J. Cameron, "Advanced coupling matrix synthesis techniques for microwave filters," IEEE Trans. Microw. Theory Tech., vol. 51, pp. 1-10, Jan. 2003.

[7] S. Bastioli, "Nonresonating mode waveguide filters," IEEE Microw. Mag., vol. 12, pp. 77-86, Dec. 2011.

[8] S. Amari, U. Rosenberg, and J. Bornemann, "Singlets, cascaded singlets and the nonresonating node model for modular design of elliptic filters," IEEE Microw. Wireless Comp. Lett., vol. 14, pp. 237-239, May 2004.

[9] S. Amari, U. Rosenberg, and R. Wu, "In-line pseudoelliptic band-reject filters with nonresonating nodes and/or phase shifts," IEEE Trans. Microw. Theory Tech., vol. 54, pp. 428-436, Jan. 2006.

[10] X. P. Chen, K. Wu, and Z. L. Li, "Dual-band and triple-band substrate integrated waveguide filters with Chebyshev and quasi-elliptic responses," IEEE Trans. Microw. Theory Tech., vol. 55, pp. 2569-2577, Dec. 2007

[11] S. Amari, U. Rosenberg, and J. Bornemann, "Adaptive synthesis and design of resonator filters with source/load-multiresonator coupling," IEEE Trans. Microw. Theory Tech., vol. 50, pp. 1969-1978, Aug. 2002.

[12] M. Salehi and E. Mehrshahi, "A closed-form formula for dispersion characteristics of fundamental SIW mode," IEEE Microw. Wireless Comp. Lett., vol. 21, no. 1, pp. 4-6, Jan. 2011. 